BMJ Open

Ophthalmology

\section{Impact of intravitreal aflibercept dosing regimens in treatment-naive patients with neovascular age-related macular degeneration in routine clinical practice in France: results from the RAINBOW study}

To cite: Weber M, Kodjikian L, Coscas F, et al. Impact of intravitreal aflibercept dosing regimens in treatment-naïve patients with neovascular age-related macular degeneration in routine clinical practice in France: results from the RAINBOW study. BMJ Open Ophthalmology 2020;5:e000377. doi:10.1136/ bmjophth-2019-000377

- Additional material is published online only. To view please visit the journal online (http://dx.doi.org/10.1136/ bmjophth-2019-000377).

For 'Presented at statement' see end of article.

Received 9 August 2019 Revised 28 January 2020 Accepted 13 February 2020

Check for updates

(c) Author(s) (or their employer(s)) 2020. Re-use permitted under CC BY-NC. No commercial re-use. See rights and permissions. Published by BMJ.

For numbered affiliations see end of article.

Correspondence to Professor Michel Weber; weber. michel@bbox.fr

\section{ABSTRACT}

Objective To evaluate 12-month outcomes in treatmentnaïve patients with neovascular (wet) age-related macular degeneration (AMD) stratified by intravitreal aflibercept (IVT-AFL) regimen.

Methods and analysis Patients included in the 12-month interim analysis of Real life of intravitreal Aflibercept In FraNce: oBservatiOnal Study in Wet AMD (RAINBOW), a 4-year, ongoing observational study conducted in France, were stratified by IVT-AFL dosing regimen. Safety $(n=593)$ and effectiveness $(n=428)$ data were analysed. Regimens included a regular cohort (three initial monthly IVT-AFL injections and $\geq 6$ injections) and irregular cohorts ( $<6$ injections) with and without three initial monthly injections. The main outcome measure was mean gain in best-corrected visual acuity (BCVA) at 12 months.

Results Mean number of IVT-AFL injections was 6.0 (all patients, $n=513$ ), 7.2 (regular cohort, $n=102$ ), 6.1 (irregular cohort with three initial monthly injections, $n=266$ ) and 5.2 (irregular cohort without three initial monthly injections, $\mathrm{n}=60$ ). Overall mean gain in BCVA at 12 months was 5 letters; +7.1 letters (regular cohort) and +5.6 letters (irregular cohort with three initial monthly injections), both $p<0.001$ versus baseline, and -1.1 letters (irregular cohort without three initial monthly injections), $\mathrm{p}=0.669$. Improvements in BCVA were also significantly greater in the regular cohort $(p<0.001)$ and irregular cohort with three initial monthly injections $(p=0.003)$ compared with the irregular cohort without three initial monthly injections. Ocular and non-ocular adverse events were reported in $14.7 \%$ and $17.4 \%$ of all patients, respectively.

Conclusion Treatment-naïve patients with neovascular AMD receiving three initial monthly injections followed by regular or irregular injections over 12 months experienced better visual acuity outcomes than those receiving irregular treatment without three initial monthly injections.

Trial registration number NCT02279537.

\section{INTRODUCTION}

Age-related macular degeneration (AMD) is a common degenerative disease of the

\section{Key messages}

What is already known about this subject? An initial analysis of the RAINBOW real-world study, reported improved visual and anatomical outcomes in treatment-naive patients with neovascular agerelated macular degeneration (AMD) treated with intravitreal aflibercept over a 12-month period.

\section{What are the new findings?}

> This real-world study shows that treatment-naïve patients with neovascular AMD receiving three initial monthly injections of intravitreal aflibercept followed by regular or irregular injection intervals over 12 months experienced better visual acuity outcomes than those receiving irregular treatment without three initial monthly injections.

\section{How might these results change the focus of} research or clinical practice?

These findings suggest that intravitreal aflibercept treatment with three initial monthly injections, followed by regular treatment, offers the best possible outcomes for patients with neovascular AMD in the real-world setting.

retina and the leading cause of legal blindness in the elderly worldwide. ${ }^{1}$ There are two forms of AMD: dry and wet. The dry form, which is characterised by drusen, pigmentary abnormalities and atrophy, accounts for $90 \%$ of cases and $10 \%$ of AMD-related blindness. $^{2}$ The wet (neovascular) form is more aggressive, and accounts for $10 \%$ of cases and $90 \%$ of AMD-related blindness and severe visual impairment. ${ }^{2}$ Neovascular AMD has a significant impact on the quality of life of patients. ${ }^{1}$ Choroidal neovascularisation (CNV) is the most common cause of vision loss due to neovascular AMD; it is triggered 
by angiogenic factors such as vascular endothelial growth factor-A (VEGF-A) ${ }^{3}$ and placental growth factor (PGF). ${ }^{24}$

The anti-VEGF agent ranibizumab is a monoclonal antibody that inhibits VEGF-A. ${ }^{5}$ Randomised studies conducted between 2003 and 2004 have demonstrated the benefits of monthly ranibizumab $(0.5 \mathrm{mg})$ dosing in patients with neovascular AMD. ${ }^{67}$ However, such frequent dosing is a burden for patients, caregivers and the healthcare system. Treatment regimens with fewer ranibizumab injections have subsequently been evaluated in studies where ranibizumab was given as needed (pro re nata $(\mathrm{PRN}))^{8}$ The Prospective OCT study with Lucentis for neovascular AMD (PrONTO Study; 2004-2005), ${ }^{9}$ study of ranibizumab in patients with subfoveal $\mathrm{CNV}$ secondary to AMD (SUSTAIN; 2006-2008) ${ }^{10}$ and Inhibition of VEGF in Age-related CNV (IVAN; 2008-2010) ${ }^{11}$ studies showed that efficacy outcomes could be achieved with less than monthly dosing. Others, such as the study of ranibizumab administered monthly or on an as-needed basis in patients with subfoveal neovascular AMD (HARBOR; 2009-2010), ${ }^{12}$ showed that a PRN regimen is slightly less favourable than a monthly dosing regimen. In a real-world setting, the ranibizumab PRN regimen was associated with undertreatment and a decline in visual acuity (VA) over 12 months. ${ }^{13-15}$

Intravitreal aflibercept (IVT-AFL) is a fusion protein that consists of human VEGF receptors 1 and 2 combined with the fragment crystallizable (Fc) portion of $\mathrm{IgG}^{5}$ It binds VEGF-A and PGF with a higher affinity than other naturally occurring VEGF receptors, so it has the potential for better efficacy. ${ }^{16}$ In the randomised VEGF Trap-Eye: Investigation of Efficacy and Safety in Wet AMD (VIEW) studies conducted between 2007 and 2011, IVTAFL $2 \mathrm{mg}$ administered every 8 weeks (2q8; after three initial monthly IVT-AFL injections) was non-inferior to the approved ranibizumab dosing schedule $(0.5 \mathrm{mg}$ every 4 weeks) in all clinical endpoints at week 52 without any marked difference in adverse events (AEs). ${ }^{17} 18$ Based on previous studies, IVT-AFL was approved with a dosing schedule in the EU of three initial monthly injections of $2 \mathrm{mg}$ followed by $2 \mathrm{q} 8$ for the first 12 months. After 12 months, injection intervals can be extended based on visual and anatomical outcomes. ${ }^{19}$ Outcomes from clinical practice settings showed that using this practical IVT-AFL dosing regimen, a 12-month visual gain of five letters or more was achieved, which is better than many previous real-world data collections. ${ }^{20-22}$ Prior to the approval of IVT-AFL in Europe in 2012, PRN dosing was the most common regimen to treat neovascular AMD in France, based on the availability of ranibizumab since 2007. Approval of IVT-AFL may have led to variations in the IVT-AFL treatment protocol/regimen used in clinical practice as physicians adapted their routine clinical practices away from the previously reactive approach. Consequently, intervals between injections may have varied substantially. In addition, we have also previously shown that not all patients receive the approved three initial doses of IVT-AFL. ${ }^{23}$
The ongoing Real life of intravitreal Aflibercept In FraNce: oBservatiOnal Study in Wet AMD (RAINBOW) study was initiated in 2014, with the objective of monitoring the outcomes achieved with IVT-AFL in treatment-naive patients with neovascular AMD treated in routine clinical practice. ${ }^{23}$ The aim of this interim analysis of the RAINBOW study was to monitor treatment outcomes in patients with neovascular AMD stratified by IVT-AFL dosing regimen (identified retrospectively) over the first 12 months of treatment: a regular cohort of patients who received the indicated posology of three initial monthly injections of IVT-AFL followed by regular injections every 2 months, with $\geq 6$ injections given during the 12 months; and two irregular cohorts who received $<6$ injections of IVT-AFL, either with or without three initial monthly injections. The aim of the stratification was to assess if there were any variations in the IVT-AFL treatment protocol and their impact on outcomes.

\section{MATERIALS AND METHODS}

Study design

RAINBOW is an ongoing, observational, multicentre, ambispective (retrospective and prospective) study to monitor the real-world effectiveness and safety of IVTAFL in treatment-naïve patients with neovascular AMD enrolled in 55 centres across France. The study began in October 2014. Data from patients who started IVTAFL treatment between 2 January and 13 October 2014 were retrospectively collected, and data from patients who started IVT-AFL treatment after 14 October 2014 were prospectively collected. This interim analysis used 12-month data collected up to 9 January 2017. However, patients will be followed for a period of 4 years or until study discontinuation.

Patients were not directly involved in the design of this study. All patients provided written informed consent to participate.

\section{Participants}

Patients aged $\geq 50$ years diagnosed with neovascular AMD and treated with IVT-AFL by their physician were eligible. Prior or current treatment with any anti-VEGF agent or macular laser in the study eye was not allowed. For patients receiving treatment only in one eye, the study eye was defined as the worst-seeing eye of each patient, and the second eye was considered as treatment naïve. For patients receiving treatment in both eyes, the first eye to be treated was considered the study eye; if both eyes were treated at the same time, the worst-seeing eye or right eye (if similar) was considered the study eye. Patients were excluded if they did not meet the indication criteria for IVT-AFL, if they had another retinal disease (diabetic retinopathy, diabetic macular oedema, myopic CNV, retinal vein occlusion, central serous chorioretinopathy, or angioid streaks), or if they were participating in any other interventional study.

\section{Outcomes}

This is an interim analysis to evaluate 12-month outcomes in treatment-naïve patients with neovascular AMD 
stratified by IVT-AFL regimen. The primary objective was to evaluate the difference in best-corrected visual acuity (BCVA), assessed preferentially by the early treatment diabetic retinopathy study (ETDRS) protocol or any visual logarithmic scale, from baseline to 12 months (range: 11-13). We converted VA readings to a standardised score using a conversion chart (online supplementary table 1).

Other assessments evaluated included the percentage of patients who gained $\geq 0, \geq 5, \geq 10$, or $\geq 15$ letters, or lost $>15$ letters at 12 months; the proportion of patients with BCVA $\geq 70$ letters at 12 months; and mean change in central retinal thickness (CRT) at month 12. All AEs occurring after the first injection of IVT-AFL (up to the 30-day period after the last IVT-AFL injection) were documented.

\section{Data sources and measurements}

For retrospectively enrolled patients, we obtained treatment(s) and outcomes from medical records. For prospectively enrolled patients, we recorded this information at routine follow-up visits. We recorded data in an electronic case report form from the inclusion date (first injection of IVT-AFL) and for up to 12 months (plus 30 days of follow-up). Each patient was identified by a unique central patient identification code.

\section{Statistical analyses}

Approximately 600 patients needed to be enrolled to achieve around 390 usable patient data sets at 48 months, with an expected precision of 1.5 letters. This included an estimated $10 \%$ loss to follow-up per year with $5 \%$ unevaluable data. We based these assumptions on the results from the VIEW studies, in which the mean (SD) difference in BCVA (letters) from baseline to 12 months was 7.9 (15.0) letters (VIEW 1) and 8.9 (14.4) letters (VIEW 2). ${ }^{18}$ The formula we used to derive these estimates was:

$$
\begin{gathered}
n=\frac{(1.96)^{2}}{i^{2}} \times S D^{2} \quad \text { where } \mathrm{i}^{2}=\text { estimated precision } \\
\sqrt{n}=1.96 \times \frac{15}{1.5}=19.6 \longrightarrow n \approx 384
\end{gathered}
$$

The study was not designed to confirm or reject any hypotheses. Interim analyses were predefined in the study protocol at yearly intervals. Statistical analyses were exploratory and descriptive in nature. We used summary statistics for categorical and quantitative (continuous) data. We described continuous data by non-missing values, mean, SD and 95\% CI. We generated frequency tables for categorical data.

All AEs were coded using the latest Medical Dictionary for Regulatory Activities V.21. We performed the analysis of the primary assessment without replacement of missing values. However, we performed two sensitivity analyses with two imputation methods for missing data (last observation carried forward (LOCF) approach, and imputation of missing data by the median value of the population). These sensitivity analyses were devised to deal with any potential bias due to premature dropouts.
The LOCF method was assumed to be more conservative as premature dropouts were expected to have poorer results than patients completing study treatment.

Safety analyses were based on data from patients who received at least one IVT-AFL injection (safety analysis set (SAS)). Patients with two treated eyes were included twice in this population. Effectiveness analyses were based on patients who also had documented assessments in the study eye at baseline and at least one follow-up visit (full analysis set (FAS)), which included one eye per patient. The primary outcome analyses were based on patients who had documented VA assessments at baseline and month 12 (FAS targeted).

Patients were retrospectively stratified by IVT-AFL dosing regimen over the first 12 months as follows: (1) regular cohort: patients who received three initial monthly IVT-AFL injections separated by 23-44 days and regular ( $\geq 6$ ) injections separated by $>39$ to $<88$ days; (2) irregular cohort with three initial monthly injections: patients who received three initial monthly IVT-AFL injections separated by 23-44 days and irregular $(<6)$ injections separated by $<39$ to $>88$ days; or (3) irregular cohort without three initial monthly injections: patients who received irregular $(<6)$ injections separated by $<39$ to $>88$ days without three initial monthly IVT-AFL injections. The three treatment cohorts evaluated in this study were derived retrospectively from the data generated within it, to assess the uptake among clinicians of the newly approved dosing regimen compared with the previous more commonly used PRN treatment modality used in the management of neovascular AMD. We compared these three treatment regimens using an analysis of variance model with treatment regimen as a fixed factor and BCVA at baseline as a covariate. The statistical analysis was performed with the software package SAS release V.9.4 (SAS Institute Inc).

\section{RESULTS}

\section{Patient disposition and baseline characteristics}

A total of 608 patients were enrolled; 593 patients (737 treated eyes) were included in the SAS and 513 patients were included in the FAS, of whom 428 comprised the FAS targeted population (see figure 1). At the time of the interim analysis, 120 patients had prematurely withdrawn. The baseline characteristics were generally similar between treatment groups (table 1).

\section{Treatments}

The mean (SD) number of IVT-AFL injections over 12 months was $6.0(2.1)$ in the FAS population $(n=513)$. A total of 428 patients had BCVA assessments at baseline and 12 months, and $368(86 \%)$ received three initial monthly IVT-AFL injections. These patients were stratified by IVTAFL regimen into the regular cohort $(n=102)$, irregular cohort with three initial monthly injections $(n=266)$ and irregular cohort without three initial monthly injections $(\mathrm{n}=60)$. The mean $(\mathrm{SD})$ number of injections was higher in the regular cohort $(7.2(0.8))$ versus the irregular 


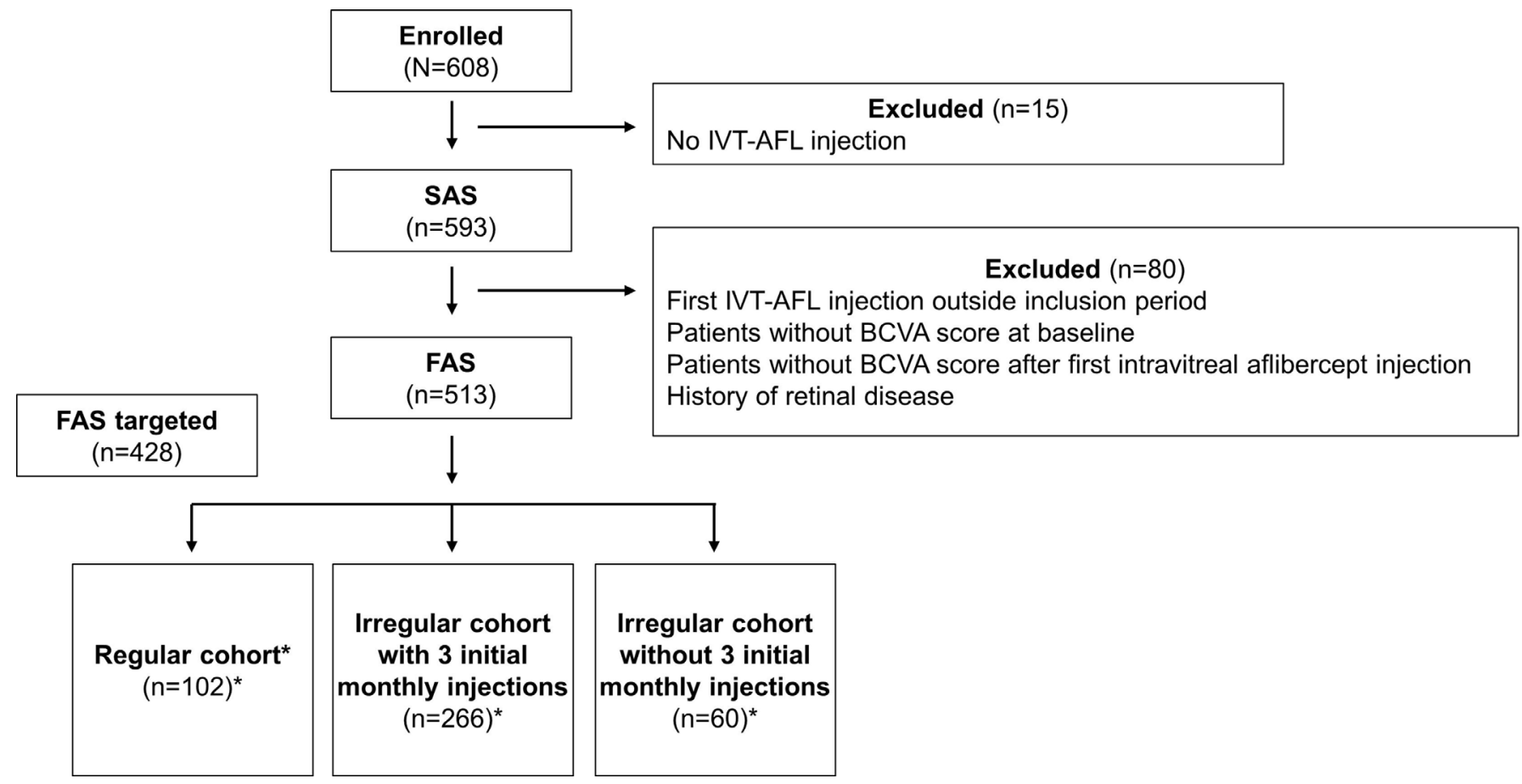

Figure 1 Patient disposition during the study. *428 patients had BCVA at baseline and 12 months (FAS targeted). BCVA, bestcorrected visual acuity; FAS, full analysis set; IVT-AFL, intravitreal aflibercept; SAS, safety analysis set.

cohort with three initial monthly IVT-AFL injections (6.1 (2.2)) and irregular cohort without three initial monthly IVT-AFL injections (5.2 (1.8)), although the duration of follow-up was comparable between the three treatment groups (online supplementary table 2 ).

\section{Visual acuity outcomes}

Mean (SD) BCVA increased from 57.5 (18.3) letters at baseline to 62.5 (18.8) letters at 12 months, resulting in a statistically significant mean (SD) gain of $5.0(16.0)$ letters in all patients $(p<0.001$ vs baseline; figure 2$)$. The results were confirmed by two sensitivity analyses conducted in the FAS population $(n=513)$.

Mean (SD) BCVA increased from 59.8 (16.5) letters at baseline to 67.0 (14.7) letters at 12 months in the regular cohort with three initial monthly injections, resulting in a mean (SD) gain of 7.1 (11.8) letters and from 56.5 (18.5) to 62.1 (18.9) letters for patients from the irregular cohort with three initial monthly injections at 12 months, resulting in a gain of 5.6 (16.4) letters (both $\mathrm{p}<0.001$ vs baseline). There was a gradual decline from baseline in mean (SD) BCVA to -1.1 (18.9) letters (from 57.6 (20.0) to 56.5 (22.9) letters) at 12 months in the irregular cohort without three initial monthly injections $(p=0.669)$. Between-group comparisons showed that the improvements in BCVA were significantly greater in the regular cohort $(p<0.001)$ and irregular cohort with three initial monthly injections $(\mathrm{p}=0.003)$ compared with the irregular cohort without three initial monthly injections (figure 2).

Gains of $\geq 15$ letters were observed in $25.2 \%$ (all patients), 22.5\% (regular cohort), 28.6\% (irregular cohort with three initial monthly injections) and $15.0 \%$ (irregular cohort without three initial monthly injections) at 12 months, respectively. Losses of $>15$ letters were observed in $6.3 \%$ (all patients), $1.0 \%$ (regular cohort), $6.8 \%$ (irregular cohort with three initial monthly injections) and $13.3 \%$ (irregular cohort without three initial monthly injections) at 12 months. At 12 months, 45.6\% (all patients), 54.9\% (regular cohort), $43.6 \%$ (irregular cohort with three initial monthly injections) and $38.3 \%$ (irregular cohort without three initial monthly injections) could read $\geq 70$ letters (figure 3 ).

\section{Anatomical outcomes}

The mean reductions in CRT from baseline to 12 months were $-110.4 \mu \mathrm{m}$ (all patients: baseline, $400.5 \mu \mathrm{m}$; 12 months, $290.1 \mu \mathrm{m}),-133.7 \mu \mathrm{m}$ (regular cohort: baseline, $400.8 \mu \mathrm{m} ; 12$ months, $267.1 \mu \mathrm{m})$ and $-113.9 \mu \mathrm{m}$ (irregular cohort with three initial monthly injections: baseline, $403.2 \mu \mathrm{m}$; 12 months, $289.3 \mu \mathrm{m}$; all $\mathrm{p}<0.001 \mathrm{vs}$ baseline). There was a smaller reduction in the irregular cohort without three initial monthly injections $(-46.2 \mu \mathrm{m}$; baseline, $381.6 \mu \mathrm{m} ; 12$ months, $335.4 \mu \mathrm{m} ; \mathrm{p}=0.036$ ) at 12 months.

\section{Safety}

Safety outcomes are summarised in table 2. One-hundredand-seventy patients (28.7\% of the treated population) declared at least one AE. In total, $287 \mathrm{AEs}$ were reported during the period of interest: 144 ocular events were experienced by $14.7 \%(\mathrm{n}=87)$ of patients and 143 nonocular events by $17.4 \%(\mathrm{n}=103)$ of patients. Thirty-five events were considered related to IVT-AFL by the investigator: 31 ocular events were experienced by $4.6 \%(n=27)$ of patients and four non-ocular events by $0.7 \%(n=4)$ of 
Table 1 Baseline demographic and clinical characteristics

\begin{tabular}{|c|c|c|c|c|}
\hline & FAS population $(n=513)$ & $\begin{array}{l}\text { Regular cohort } \\
(n=102)\end{array}$ & $\begin{array}{l}\text { Irregular cohort with } \\
\text { treatment initiation }(n=266)\end{array}$ & $\begin{array}{l}\text { Irregular cohort without } \\
\text { treatment initiation }(n=60)\end{array}$ \\
\hline Age, years* & $79.6(7.9)$ & $79.2(7.6)$ & $79.2(7.7)$ & $81.3(7.1)$ \\
\hline Male, $\mathrm{n}(\%)$ & $199(38.8)$ & $41(40.2)$ & $103(38.7)$ & $19(31.7)$ \\
\hline \multicolumn{5}{|l|}{ Neovascular AMD diagnosis, $\mathrm{n}(\%)$} \\
\hline One eye & $513(100.0)$ & $102(100.0)$ & $266(100.0)$ & $60(100.0)$ \\
\hline Duration of neovascular AMD, months $†$ & 0.1 & 0.1 & 0.2 & 0.1 \\
\hline \multicolumn{5}{|l|}{ Comorbidities, n (\%) } \\
\hline Diabetes mellitus & $42(8.2)$ & $7(6.9)$ & $21(7.9)$ & $8(13.3)$ \\
\hline Hypertension & $190(37.0)$ & $38(37.3)$ & $106(39.8)$ & $19(31.7)$ \\
\hline Cardiovascular disease & $85(16.6)$ & $14(13.7)$ & $47(17.7)$ & $8(13.3)$ \\
\hline CRT, $\mu \mathrm{m}(\mathrm{n}=458)$ & $399.4(141.9)$ & $403.9(147.8)$ & $402.9(140.1)$ & $380.4(148.4)$ \\
\hline \multicolumn{5}{|l|}{ CNV on FA, $n(\%)(n=386)$} \\
\hline Minimally classic & $34(8.8)$ & $11(12.5)$ & $14(7.0)$ & $6(13.6)$ \\
\hline Predominantly classic & $76(19.7)$ & $22(25.0)$ & $29(14.6)$ & $10(22.7)$ \\
\hline Purely occult & $130(33.7)$ & $34(38.6)$ & $62(31.2)$ & $15(34.1)$ \\
\hline Retinal choroidal anastomosis & $43(11.1)$ & $6(6.8)$ & $25(12.6)$ & $7(15.9)$ \\
\hline RPE detachment & $31(8.0)$ & $5(5.7)$ & $23(11.6)$ & $1(2.3)$ \\
\hline Extrafoveal & $3(0.8)$ & $1(1.1)$ & $2(1.0)$ & 0 \\
\hline Subfoveal & $20(5.2)$ & $2(2.3)$ & $13(6.5)$ & $1(2.3)$ \\
\hline Presence of RPE tear, $n(\%)(n=386)$ & $7(1.8)$ & 0 & $4(2.0)$ & $2(4.5)$ \\
\hline \multicolumn{5}{|l|}{ Outcomes on SD OCT, n (\%) ( $\mathrm{n}=489)$} \\
\hline Presence of subretinal fluid & $403(82.4)$ & $80(81.6)$ & $213(83.5)$ & $39(69.6)$ \\
\hline Presence of intraretinal fluid & $309(63.2)$ & $58(59.2)$ & $159(62.4)$ & $38(67.9)$ \\
\hline Presence of sub-RPE fluid & $241(49.3)$ & $47(48.0)$ & $134(52.5)$ & $28(50.0)$ \\
\hline Pigment epithelium detachment & 309 (63.2) & $52(53.1)$ & $175(68.6)$ & $31(55.4)$ \\
\hline Suspicion for RAP & $59(12.1)$ & $13(13.3)$ & $31(12.2)$ & 7 (12.5) \\
\hline PCV & $31(6.3)$ & $6(6.1)$ & $14(5.5)$ & $5(8.9)$ \\
\hline
\end{tabular}

${ }^{*}$ Mean (SD).

†Median.

AMD, age-related macular degeneration; CNV, choroidal neovascularisation; CRT, central retinal thickness; FA, fluorescein angiography; FAS, full analysis set; PCV, polypoidal choroidal vasculopathy; RAP, retinal angiomatous proliferation; RPE, retinal pigment epithelium; SD OCT, spectral domain optical coherence tomography.

patients. Twenty-three ocular events and 17 non-ocular events were responsible for a treatment discontinuation in 31 patients; 17 of these events, all ocular, were considered to be related to the treatment according to the physician. A total of 60 serious AEs occurred in $7.8 \%$ $(n=46)$ of patients when treated with IVT-AFL and one patient presented one serious $\mathrm{AE}$ when receiving other treatments. Overall, the most common treatment-related ocular AEs were vitreous floaters (1.9\% of patients), lack of effectiveness $(1.3 \%)$, eye pain $(1.2 \%)$ and increased lacrimation $(1.2 \%)$. Four patients died during the follow-up period.

\section{DISCUSSION}

The ongoing RAINBOW study is monitoring resource use and outcomes with IVT-AFL in treatment-naïve patients with neovascular AMD in France. The aim of this interim analysis was to monitor 12-month outcomes by evaluating three different IVT-AFL regimens. Patients were treated in accordance with the investigator's normal clinical practice; these patients were then retrospectively divided into three cohorts based on the treatment protocol utilised (as described). Although some variability in the IVT-AFL treatment protocol was observed in this real-world setting, of the 593 patients who received at least one injection of IVT-AFL, 368 of $428(86 \%)$ received three initial monthly IVT-AFL injections. Variability in the treatment protocols used with IVT-AFL is likely due to the option of anti-VEGF administration using either a reactive $(\mathrm{PRN})$ or proactive treatment approach. Although both approaches incorporate three initial monthly injections, our observation that this does not occur in all patients, is not unique and has been seen previously with IVT-AFL and ranibizumab (with both a fixed-dose or treat-and-extend dosing). ${ }^{23-25}$ This, therefore, provides the rationale to evaluate the impact of any differences in treatment protocols on patient outcomes in clinical practice. 


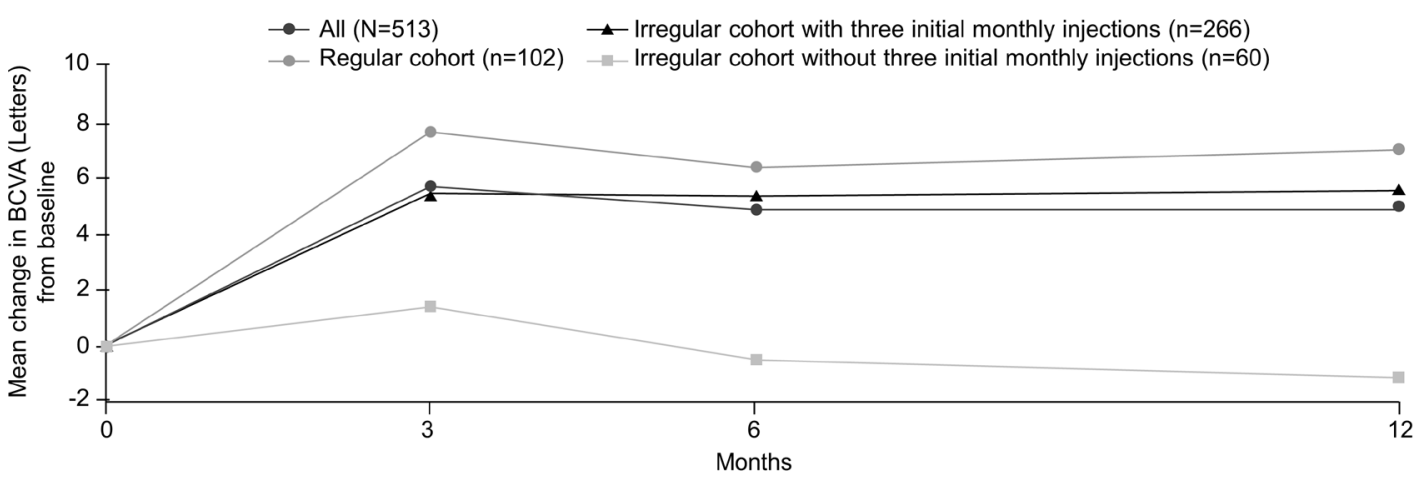

\begin{tabular}{|l|c|c|c|}
\hline & $\begin{array}{c}\text { Mean (SD) } \\
\text { BCVA score } \\
\text { at baseline }\end{array}$ & $\begin{array}{c}\text { Mean (SD) } \\
\text { BCVA score } \\
\text { at 12 months }\end{array}$ & $\begin{array}{c}\text { Mean (SD) [95\% Cl] } \\
\text { change in letters } \\
\text { at 12 months }\end{array}$ \\
\hline All $(\mathrm{N}=513)$ & $57.5(18.3)$ & $62.5(18.8)$ & $+5.0(16.0)[3.5-6.5]^{*}$ \\
\hline Regular cohort $(n=102)$ & $59.8(16.5)$ & $67.0(14.7)$ & $+7.1(11.8)[4.8-9.4)^{* \dagger}$ \\
\hline Irregular cohort with three initial monthly injections $(n=266)$ & $56.5(18.5)$ & $62.1(18.9)$ & $+5.6(16.4)[3.6-7.6)^{*}$ \\
\hline Irregular cohort without three initial monthly injections $(n=60)$ & $57.6(20.0)$ & $56.5(22.9)$ & $-1.1(18.9)[-5.9-3.8]$ \\
\hline
\end{tabular}

Figure 2 Mean change in BCVA (letters) from baseline to 12 months by IVT-AFL regimen. All: $n=375$ (month 3); $n=402$ (month 6). Regular cohort: $n=69$ (month 3); $n=87$ (month 6). Irregular cohort with three initial monthly injections: $n=216$ (month 3); $n=215$ (month 6). Irregular cohort without three initial monthly injections: $n=39$ (month 3); $n=52$ (month 6). ${ }^{*} p<0.001$ versus baseline, ${ }^{\dagger} p<0.001$ versus irregular cohort without three initial monthly injections; ${ }^{\ddagger} p=0.003$ versus irregular cohort without three initial monthly injections. BCVA, best-corrected visual acuity; IVT-AFL, intravitreal aflibercept.

In the pooled analysis of the VIEW studies, the mean change in BCVA was 8.4 letters, the proportion of patients gaining $\geq 15$ letters was $30.9 \%$ and the percentage of patients maintaining VA (losing $<15$ letters) was $95.3 \%$ at month 12 after a mean of 7.5 IVT-AFL injections in the combined IVT-AFL 2q8 group after three initial monthly injections. ${ }^{17} 18$ In this study, the overall results showed a mean visual gain of five letters, and were closer to those seen in the VIEW studies when the regimen included three monthly injections and a regular follow-up with a gain of 7.1 letters. Furthermore, the secondary endpoints of our entire cohort were very similar to the ones of the VIEW studies, with gains of $\geq 15$ letters observed in $25.2 \%$ of patients and losses of $>15$ letters observed in only $6.3 \%$. When examining BCVA at 12 months for the overall population (62.5 letters), it is noticeable how similar this is to those values observed at 12 months in the VIEW 1 (63.6 letters) and VIEW 2 (60.5 letters) studies.

Furthermore, the present results were similar to other real-world series using IVT-AFL as first-line therapy. In

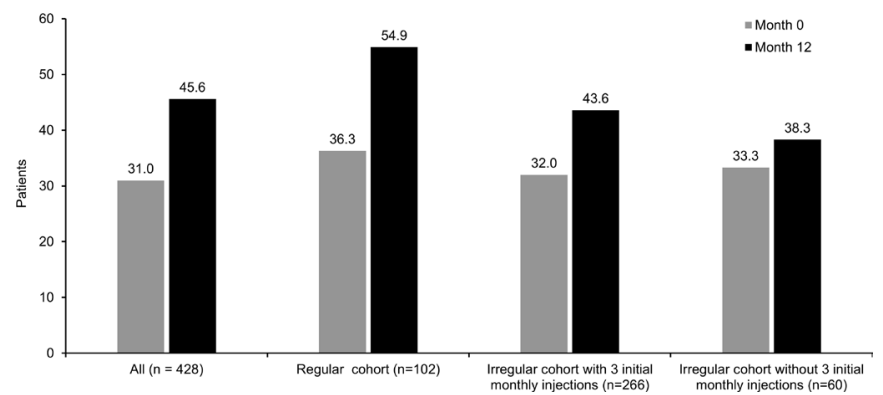

Figure 3 Patients with BCVA $\geq 70$ letters at 12 months by IVT-AFL regimen. Observed analysis. BCVA, best-corrected visual acuity; IVT-AFL, intravitreal aflibercept. a retrospective real-world analysis of 1840 treatmentnaive eyes diagnosed with neovascular AMD using the same IVT-AFL treatment protocol that was used in the VIEW studies, the mean BCVA gain at 12 months was 5.1 letters, with a mean of seven IVT-AFL injections. ${ }^{22}$ Similar improvements in BCVA were reported in another retrospective real-world analysis conducted in the United Kingdom: 109 eyes of 102 patients diagnosed with neovascular AMD and treated with aflibercept were analysed (fixed dosing year 1 followed by a treat-and-extend approach). The mean BCVA gain (letters) was 5.9 (year 1), 6.4 (year 2) and 6.6 (year 3), and the corresponding median number of injections was 8 in year 1 , and 4 in years 2 and $3 .^{20}$ A prospective analysis (prospective non-interventional study to assess the effectiveness of aflibercept in routine clinical practice in patients with neovascular AMD (PERSEUS)) conducted in Germany indicated that outcomes in treatment-naïve patients were also more favourable in those receiving regular IVT-AFL injections (mean VA gain: 8.0 letters) than the irregularly treated population at 12 months (mean VA gain: 4.0 letters), ${ }^{26}$ highlighting the need for consistent and regular treatment regimens to obtain optimal outcomes as seen in clinical studies.

Variations were observed in the administration of IVT-AFL treatment in France, which differed from the indicated treatment regimen. One possible explanation is that prior to the approval of IVT-AFL, PRN was the most common regimen to treat neovascular AMD in France, and some physicians may have continued to use this regimen. Of note, phase 3 studies have not assessed the efficacy of IVT-AFL without the three indicated initial monthly injections. Our study did not assess the reasons for the lack 
Table 2 Safety outcomes at 12 months

\begin{tabular}{|c|c|c|c|c|}
\hline AE, n (\%) & SAS $(n=593)$ & $\begin{array}{l}\text { Regular cohort } \\
(n=102)\end{array}$ & $\begin{array}{l}\text { Irregular cohort } \\
\text { with treatment } \\
\text { initiation }(n=266)\end{array}$ & $\begin{array}{l}\text { Irregular cohort } \\
\text { without treatment } \\
\text { initiation }(n=60)\end{array}$ \\
\hline Any AE & $170(28.7)$ & $22(21.6)$ & $77(28.9)$ & $12(20.0)$ \\
\hline Ocular & $87(14.7)$ & $15(14.7)$ & $48(18.0)$ & $4(6.7)$ \\
\hline Non-ocular & $103(17.4)$ & $11(10.8)$ & $42(15.8)$ & $8(13.3)$ \\
\hline Any treatment-related $\mathrm{AE}$ & $31(5.2)$ & $5(4.9)$ & $17(6.4)$ & $1(1.7)$ \\
\hline Ocular & $27(4.6)$ & $4(3.9)$ & $16(6.0)$ & $1(1.7)$ \\
\hline Non-ocular & $4(0.7)$ & $1(1.0)$ & $1(0.4)$ & 0 \\
\hline \multicolumn{5}{|c|}{ Most common $(>1 \%)$ treatment-related ocular AEs occurring in any treatment group* } \\
\hline Vitreous floaters & $11(1.9)$ & $3(2.9)$ & $6(2.3)$ & - \\
\hline Drug ineffective & $8(1.3)$ & $1(1.0)$ & $5(1.9)$ & - \\
\hline Eye pain & $7(1.2)$ & - & $4(1.5)$ & $1(1.7)$ \\
\hline Lacrimation increased & $7(1.2)$ & $2(2.0)$ & $5(1.9)$ & - \\
\hline Metamorphopsia & $6(1.0)$ & $1(1.0)$ & $3(1.1)$ & - \\
\hline Visual acuity reduced & $5(0.8)$ & $1(1.0)$ & $3(1.1)$ & - \\
\hline Vision blurred & $5(0.8)$ & $1(1.0)$ & $3(1.1)$ & - \\
\hline Eye allergy & $5(0.8)$ & $1(1.0)$ & - & $1(1.7)$ \\
\hline Injection-site pain & $4(0.7)$ & - & $3(1.1)$ & - \\
\hline Photophobia & - & - & $3(1.1)$ & - \\
\hline Choroidal neovascularisation & - & - & - & $1(1.7)$ \\
\hline Retinal oedema & - & - & - & $1(1.7)$ \\
\hline Retinal vein occlusion & - & - & - & $1(1.7)$ \\
\hline Any treatment-emergent SAE & $46(7.8)$ & $7(6.9)$ & $18(6.8)$ & $4(6.7)$ \\
\hline Ocular & $4(0.7)$ & $2(2.0)$ & $2(0.8)$ & 0 \\
\hline Non-ocular & $42(7.1)$ & $5(4.9)$ & $16(6.0)$ & $4(6.7)$ \\
\hline \multicolumn{5}{|l|}{ Treatment-related ocular SAE } \\
\hline Traumatic cataract & $1(0.2)$ & $1(1.0)$ & - & - \\
\hline \multicolumn{5}{|l|}{ Treatment-related non-ocular SAE } \\
\hline Transient ischaemic attack & $4(0.7)$ & - & $3(1.1)$ & - \\
\hline Peripheral arterial occlusive disease & $1(0.2)$ & - & - & - \\
\hline Any AE leading to discontinuation & $31(5.2)$ & $4(3.9)$ & $17(6.4)$ & $1(1.7)$ \\
\hline Ocular & $20(3.4)$ & $2(2.0)$ & $12(4.5)$ & $1(1.7)$ \\
\hline Non-ocular & $13(2.2)$ & $2(2.0)$ & $7(2.6)$ & 0 \\
\hline Any treatment-related $\mathrm{AE}$ leading to discontinuation & $14(2.4)$ & $1(1.0)$ & $8(3.0)$ & $1(1.7)$ \\
\hline Ocular & $14(2.4)$ & $1(1.0)$ & $8(3.0)$ & $1(1.7)$ \\
\hline Non-ocular & $0(0.0)$ & 0 & 0 & 0 \\
\hline Death (not treatment-related) & $4(0.7)$ & 0 & 0 & 0 \\
\hline
\end{tabular}

Values in the cohort columns are based on the FAS population.

${ }^{*} \mathrm{AE}$ rates $<0.5 \%$ are not shown.

AE, adverse event; FAS, full analysis set; SAE, serious adverse event; SAS, safety analysis set.

of compliance with the initial threemonthly injections; however, a lack of adherence to, or persistence with, antiVEGF therapy has been evaluated in other studies. These have identified various factors including system-related factors such as difficulties in planning monthly injections or scheduling patient visits, as well as patient and diseaserelated characteristics. For example, a multinational study reported that patients missed clinic visits as the caregiver was unable to take them to the appointment, because of fear about receiving an injection, or because of illness. ${ }^{27} \mathrm{~A}$ study in France reported patients frequently missed treatment appointments over the summer vacation period, to the detriment of outcomes, and highlighted the importance of explaining to patients the need for treatment adherence, despite their need for holidays. ${ }^{28}$

Other real-world studies reported the results of treatment with ranibizumab in patients with neovascular AMD in Europe, using a PRN regimen in the years 2006-2011. In LUMIERE, an observational study of ranibizumab use in French clinical practice, conducted from 2006 to 2009, 
the mean BCVA change from baseline to month 12 was 3.2 letters in patients receiving a mean of 5.1 injections, ${ }^{15}$ with fewer than $40 \%$ of patients receiving the recommended treatment of three initial monthly injections, suggesting that failure to follow the recommended dosing was associated with lower than anticipated efficacy. In TWIN, a follow-up conducted from 2010 to $2011,56.6 \%$ of patients received the recommended treatment of three initial monthly injections (mean of 5.6 ranibizumab injections over 12 months), with a slightly better improvement in VA (gain of 4.3 letters) ${ }^{29}$ These studies, like ours, reinforce the importance of following the approved treatment protocol of three initial monthly IVT-AFL injections.

Analysis of UK medical records to compare the effectiveness of IVT-AFL in proactive regimens (fixed or treat-and-extend) versus ranibizumab in reactive regimen (PRN) found that patients treated with IVT-AFL achieved greater VA gains at 1 year (+6.1 ETDRS letters) than those treated with ranibizumab $(+1.6$ ETDRS letters, $p=2.2 \mathrm{e}-16)$. The authors attributed the observed difference to the dosing regimen rather than the anti-VEGF agent used. However, it should be noted that, although the IVT-AFL group had significantly more injections than the ranibizumab group (7.0 vs $5.8 ; \mathrm{p}<2.2 \mathrm{e}-16)$, they required fewer clinic visits (9.0 vs 10.8 ; $\mathrm{p}<2.2 \mathrm{e}-16) .{ }^{30}$

The safety profile of IVT-AFL in the real-life RAINBOW study was consistent with that reported in previous studies. Of the 31 patients with treatment-related AEs, three were serious (transient ischaemic attack, peripheral arterial occlusive disease and traumatic cataract). Four deaths were recorded; none were considered related to IVTAFL. No new safety signal was seen.

There are some limitations to this observational study; the visit schedule was at the discretion of the treating physician, which resulted in missing data at time points throughout the study. To address this, we included some flexibility around the initial dosing period, dosing intervals and final BCVA assessment. In addition, two sensitivity analyses were conducted with two different imputation methods for missing values (LOCF and median); the results remained consistent across these analyses. This interim analysis was also exploratory, and the results should be interpreted with caution; notably, allocation to treatment schedules was not randomised and no analysis of disease severity that could influence treatment regimen was carried out. However, noting the earlier limitations, given that the data sources were from 55 ophthalmological centres utilising real-world practice, our overall findings could be generalised to wider practice.

In conclusion, this second interim analysis of the RAINBOW study confirms the preliminary results, ${ }^{23}$ which demonstrated that IVT-AFL was associated with visual and anatomical improvements over 12 months under realworld conditions in France. Our results emphasise the benefit that treatment-naïve patients with neovascular AMD obtained with a first-line treatment with IVT-AFL in real life, with a clinically meaningful mean VA gain of five letters at 1 year, and even greater gains when three initial monthly injections were given. Thus, even though the study was not randomised, the results suggest that better results can be obtained with a regular treatment in accordance with the summary of product characteristics (ie, three initial injections within 23-44 days and further injections spaced by no more than 88 days) for the first year. Results from this study should help physicians understand the overall importance of three initial monthly IVT-AFL injections followed by regular treatment during the first year with the goal of maximising patient outcomes.

Author affiliations

${ }^{1} \mathrm{CHU}$ Hôtel-Dieu, Nantes, France

${ }^{2}$ Centre Hospitalier de la Croix Rousse, Lyon, France

${ }^{3}$ Centre Odéon, Paris, France

${ }^{4}$ Clinique Saint-Martin, Ramsay Générale de Santé, Caen, France

${ }^{5}$ Centre Ophtalmologique des Arceaux, Montpellier, France

${ }^{6}$ Bayer Healthcare SAS, Loos, France

${ }^{7}$ Centre d'Imagerie et de Laser, Paris, France

Presented at

Annual Meeting of the Association for Research in Vision and Ophthalmology, Seattle, Washington, USA, 1-5 May 2016; 16th European Society of Retina Specialists Congress, Copenhagen, Denmark, 8-11 September 2016; 2017 MaculArt Meeting: Imaging and Managing Macular Diseases, Paris, France, 2-4 July 2017; 123rd Congrès de la Société Française d'Ophtalmologie, France, Paris, 5-9 May 2017; 49th Annual Scientific Congress of the Royal Australian and New Zealand College of Ophthalmologists, Perth, WA, Australia, 28 0ctober-1 November 2017; 9th Annual Congress on Controversies in Ophthalmology: Europe, Athens, Greece, 22-24 March 2018; 124th Congrès de la Société Française d'Ophtalmologie, Paris, France, 5-8 May 2018.

Acknowledgements The authors wish to thank the RAINBOW study investigators:Professor Cohen, Dr Simon, Dr Donati, Dr Krivosic, Dr Mauget Faysse, Dr Queguiner, Dr Navarre, Dr Li Calzi, Dr Bousquet, Dr Chiambaretta, Dr NghiemBuffet, Professor Creuzot Garcher, Dr Aknin, Professor Weber, Dr Bruneau, Dr Tourame de Massiac, Dr Boulet, Dr Danan Husson, Dr Lehmann, Dr Rousseau, Dr Fajnkuchen, Dr Uzzan, Dr Mohand Said, Dr Lebreton, Dr Jeguirim, Professor Arndt, Dr Briend Joulain, Dr Gonzalez, Dr Matonti, Dr Sillaire Houtmann, Dr Quentel, Dr Lecleire-Collet, Dr Girmens, Dr Masse, Dr Scemmama, Dr Benisty, Dr Robinet Combes, Professor Dot, Dr Courjaret, Dr Dumas, Dr Parier, Dr Delhay, Dr Ullerin, Professor Baillif, Dr Ducos de Lahitte, Dr Garcia, Dr Gambrelle, Dr Agard, Dr Joannot, Dr Blanchard, Dr Le Tien, Professor Quintyn, Dr Becquet, Dr Asrarguis, Dr Velasque, Dr Afriat, Dr Ramahefasolo, Dr El Chehab, Dr George, Dr Verdier, Dr Kuhn, Professor Kodjikian, Dr Leal, Dr Rosier, Professor Milazzo, Dr Kirsch, Dr Russo, Dr Pecheur, Professor Chiquet, Dr Aubry Quenet, Dr Salame, Dr Bentaleb, Dr Colasse Marthelot, Dr Dominguez, Dr Benzerroug, Dr Khaitrine, Dr Alfonsi, Dr Simoni, Dr Aptel, Dr Serny, Professor Korobelnik, Dr Mathis, Dr Quaranta-El-Maftouhi, Dr Fourmaux, Dr Drimbea, Dr Guigui, Dr Paoli, Dr Sagnan, Dr Zhou, Dr Coscas, Dr Combillet, Dr Jankowski, Professor Saleh, Dr Oubraham, Dr Atmani, Dr Martinet, Dr Chibani, Dr Chobeaux, Dr Hubanova, Dr Francais, Professor Delyfer, Professor Tadayoni, Professor Guigou, Professor Leveziel, Dr Allieu, Dr Sagnieres, Dr Tran, Dr Massamba, Dr Favard, Dr Rougier, Dr Erginay, Dr Marc, Dr Boissonnot, Dr Rohart, Dr Zeghidi, Professor Soubrane, Dr Krawczyk. Medical and scientific coordination were performed by Armelle Baillou-Beaufils, Head of Ophthalmology Department (Bayer France) and Ingrid Dufour, Clinical Research and Medical Advisor (Bayer France). Operational coordination was performed by Anne Marie Delaunay, Head of PMS Group (Bayer France).

Contributors MW, LK, FC, CF, IA, ID and SYC contributed to the design; data acquisition, analysis and interpretation; and preparation and final review of the manuscript.

Funding The RAINBOW study was sponsored by Bayer HealthCare SAS. The sponsor participated in the design and conduct of the study, analysis of the data and preparation of the manuscript. Medical writing assistance was provided by Nathan Ley, PhD, of PAREXEL and Louise Brady, PhD, of Apothecom, and was funded by Bayer HealthCare SAS.

Competing interests MW: Alcon, Alimera, Allergan, Bayer, Novartis, Thea. LK: AbbVie, Allergan, Bayer, Novartis, Roche, Thea. FC: Allergan, Bayer, Novartis, Roche. 
CF: Allergan, Bayer, Novartis; IA: Bayer, Novartis. ID: employee of Bayer. SYC: Allergan, Bayer, Novartis, Thea, Tilak.

Patient and public involvement Patients and/or the public were not involved in the design, or conduct, or reporting or dissemination plans of this research. Refer to the Methods section for further details.

\section{Patient consent for publication Not required.}

Ethics approval No independent ethics committee and institutional review board approval was obtained as this is not required in France for observational studies. However, the RAINBOW study protocol was reviewed and approved by a French data privacy committee (Comité Consultatif sur le Traitement de l'Information en Matière de Recherche dans le Domaine de la Santé, and Commission Nationale de I'Informatique et des Libertés).

Provenance and peer review Not commissioned; externally peer reviewed.

Data availability statement Availability of the data underlying this publication will be determined according to Bayer's commitment to the EFPIA/PhRMA "Principles for responsible clinical trial data sharing". This pertains to scope, time point and process of data access. As such, Bayer commits to sharing upon request from qualified scientific and medical researchers patients-level clinical trial data, study-level clinical trial data, and protocols from clinical trials in patients for medicines and indications approved in the United States (US) and European Union (EU) as necessary for conducting legitimate research. This applies to data on new medicines and indications that have been approved by the EU and US regulatory agencies on or after January 01, 2014. Interested researchers can use to request access to anonymized patient-level data and supporting documents from clinical studies to conduct further research that can help advance medical science or improve patient care. Information on the Bayer criteria for listing studies and other relevant information is provided in the Study sponsors section of the portal. Data access will be granted to anonymized patient-level data, protocols and clinical study reports after approval by an independant scientific review panel. Bayer is not involved in the decisions made by the independant review panel. Bayer will take all necessary measures to ensure that patient privacy is safeguarded.

Open access This is an open access article distributed in accordance with the Creative Commons Attribution Non Commercial (CC BY-NC 4.0) license, which permits others to distribute, remix, adapt, build upon this work non-commercially, and license their derivative works on different terms, provided the original work is properly cited, appropriate credit is given, any changes made indicated, and the use is non-commercial. See: http://creativecommons.org/licenses/by-nc/4.0/.

ORCID iD

Michel Weber http://orcid.org/0000-0001-5884-1177

\section{REFERENCES}

1 Gehrs KM, Anderson DH, Johnson LV, et al. Age-related macular degeneration--emerging pathogenetic and therapeutic concepts. Ann Med 2006;38:450-71.

2 Quillen DA. Common causes of vision loss in elderly patients. Am Fam Physician 1999;60:99-108.

3 Ablonczy Z, Dahrouj M, Marneros AG. Progressive dysfunction of the retinal pigment epithelium and retina due to increased VEGF-A levels. Faseb J 2014;28:2369-79.

4 Huo X, Li Y, Jiang Y, et al. Inhibition of ocular neovascularization by co-inhibition of VEGF-A and PIGF. Cell Physiol Biochem 2015;35:1787-96

5 Papadopoulos N, Martin J, Ruan Q, et al. Binding and neutralization of vascular endothelial growth factor (VEGF) and related ligands by VEGF trap, ranibizumab and bevacizumab. Angiogenesis 2012;15:171-85.

6 Brown DM, Kaiser PK, Michels M, et al. Ranibizumab versus verteporfin for neovascular age-related macular degeneration. $N$ Engl J Med 2006;355:1432-44.

7 Rosenfeld PJ, Brown DM, Heier JS, et al. Ranibizumab for neovascular age-related macular degeneration. N Engl J Med 2006;355:1419-31.

8 Lanzetta P, Loewenstein A, Vision Academy Steering Committee. Fundamental principles of an anti-VEGF treatment regimen: optimal application of intravitreal anti-vascular endothelial growth factor therapy of macular diseases. Graefes Arch Clin Exp Ophthalmol 2017;255:1259-73.

9 Lalwani GA, Rosenfeld PJ, Fung AE, et al. A variable-dosing regimen with intravitreal ranibizumab for neovascular age-related macular degeneration: year 2 of the PrONTO study. Am J Ophthalmol 2009;148:43-58.

10 Holz FG, Amoaku W, Donate J, et al. Safety and efficacy of a flexible dosing regimen of ranibizumab in neovascular age-related macular degeneration: the SUSTAIN study. Ophthalmology 2011;118:663-71.

11 Chakravarthy U, Harding SP, Rogers CA, et al. Ranibizumab versus bevacizumab to treat neovascular age-related macular degeneration: one-year findings from the IVAN randomized trial. Ophthalmology 2012;119:1399-411.

12 Busbee BG, Ho AC, Brown DM, et al. Twelve-month efficacy and safety of $0.5 \mathrm{Mg}$ or $2.0 \mathrm{Mg}$ ranibizumab in patients with subfoveal neovascular age-related macular degeneration. Ophthalmology 2013;120:1046-56.

13 Gerding H, Loukopoulos V, Riese J, et al. Results of flexible ranibizumab treatment in age-related macular degeneration and search for parameters with impact on outcome. Graefes Arch Clin Exp Ophthalmol 2011;249:653-62.

14 Holz FG, Tadayoni R, Beatty S, et al. Multi-country real-life experience of anti-vascular endothelial growth factor therapy for wet age-related macular degeneration. Br J Ophthalmol 2015;99:220-6.

15 Cohen SY, Mimoun G, Oubraham H, et al. Changes in visual acuity in patients with wet age-related macular degeneration treated with intravitreal ranibizumab in daily clinical practice: the LUMIERE study. Retina 2013;33:474-81.

16 Heier J. Vegf Trap-Eye for exudative AMD, retinal physician. Ambler, PA: PentaVision, 2009. Available: https://www.retinalphysician.com/ issues/2009/april-2009/vegf-trap-eye-for-exudative-amd

17 Schmidt-Erfurth U, Kaiser PK, Korobelnik J-F, et al. Intravitreal aflibercept injection for neovascular age-related macular degeneration: ninety-six-week results of the view studies. Ophthalmology 2014;121:193-201.

18 Heier JS, Brown DM, Chong V, et al. Intravitreal aflibercept (VEGF trap-eye) in wet age-related macular degeneration. Ophthalmology 2012;119:2537-48.

19 Bayer. Eylea (aflibrecept) summary of product characteristics, 2018. Available: https://wwwemaeuropaeu/documents/productinformation/eylea-epar-product-information enpdf

20 Eleftheriadou M, Gemenetzi M, Lukic M, et al. Three-Year outcomes of aflibercept treatment for neovascular age-related macular degeneration: evidence from a clinical setting. Ophthalmol Ther 2018;7:361-8.

21 Eleftheriadou M, Vazquez-Alfageme C, Citu CM, et al. Long-Term outcomes of aflibercept treatment for neovascular age-related macular degeneration in a clinical setting. Am J Ophthalmol 2017:174:160-8.

22 Talks JS, Lotery AJ, Ghanchi F, et al. First-Year visual acuity outcomes of providing aflibercept according to the view study protocol for age-related macular degeneration. Ophthalmology 2016;123:337-43.

23 Weber M, Velasque L, Coscas F, et al. Effectiveness and safety of intravitreal aflibercept in patients with wet age-related macular degeneration treated in routine clinical practices across France: 12-month outcomes of the rainbow study. BMJ Open Ophthalmol 2019;4:e000109.

24 Framme C, Eter N, Hamacher T, et al. Aflibercept for patients with neovascular age-related macular degeneration in routine clinical practice in Germany. Ophthalmol Retina 2018;2:539-49.

25 Johnston RL, Carius H-J, Skelly A, et al. A retrospective study of ranibizumab treatment regimens for neovascular age-related macular degeneration (nAMD) in Australia and the United Kingdom. Adv Ther 2017:34:703-12.

26 Framme C, Eter N, Hamacher T, et al. Aflibercept for patients with neovascular age-related macular degeneration in routine clinical practice in Germany: Twelve-Month outcomes of PERSEUS. Ophthalmol Retina 2018;2:539-49.

27 Varano M, Eter N, Winyard S, et al. Current barriers to treatment for wet age-related macular degeneration (WAMD): findings from the wAMD patient and caregiver survey. Clin Ophthalmo 2015:9:2243-50.

28 Massamba N, Dirani A, Knoeri J, et al. Evaluating the impact of summer vacation on the visual acuity of AMD patients treated with ranibizumab. Eye 2015;29:1453-7.

29 Souied EH, Oubraham H, Mimoun G, et al. Changes in visual acuity in patients with wet age-related macular degeneration treated with intravitreal ranibizumab in daily clinical practice: the twin study. Retina 2015;35:1743-9.

30 Lee AY, Lee CS, Egan CA, et al. Uk AMD/DR EMR report IX: comparative effectiveness of predominantly as needed (PRN) ranibizumab versus continuous aflibercept in UK clinical practice. $\mathrm{Br}$ J Ophthalmol 2017;101:1683-8. 